\title{
XXIX.
}

Aus dem pharmakologisehen Laboratorium der Universität von Miehigan in Ann Arbor, U. S. A.

\section{Ueber das Ricinusgift.}

Von

Arthur R. Cushny.

Dass die Ricinussamen ein heftiges Gift enthalten, ist schon lange bekannt, und eine ganze Reihe von Forsehern hat versucht, daś giftige Prineip rein darzutellen. Die ältere Litteratur ist in der Arbeit von Stillmark und Kobert ${ }^{1}$ ) aufgeführt, und ich kann die Angabe derselben darum hier unterlassen. Die neuere Litteratur fängt mit 1883 an, als die Untersuchung des Ricinusgiftes in dem Institute für experimentelle Pharmakologie zu Strassburg als Thema aufgenommen und zuerst von Bubnow bearbeitet wurde. Seine Resultate sind aber wegen seines frühen Todes nicht genau bekannt geworden. Er scheint (nach Dixson) einen mit Salzsäure bereiteten Auszug der Ricinussamen mit Alkali gefällt und diesen giftigen Niedersehlag weiter bearbeitet zu haben.

Später nahm Dixson, ebenfalls im pharmakologisehen Institut zu Strassburg, die Untersuchung wieder auf. Er fand 2), dass ein salzsaurer Auszug, mit kohlensaurem Natron versetzt, einen giftigen Niederschlag giebt, dass man aber durch Fällen eines wässerigen Auszuges mit Alkohol eine bessere Ausbeute erhält. Dieser Niederschlag enthielt viel Eiweiss, sowie ein Glykosid. Alle Versuche, ein reineres giftiges Product durch fractionirte Fällung mit Alkohol zu erzielen, blieben fruchtlos, indem nach wiederholter Fällung das Präeipität am Ende ganz wirkungslos wurde. Ein etwas reineres Präparat erhielt er dureh Versetzen des wässerigen Auszuges mit Blei-

1) Arbeiten des Pharmakologischen Instituts zu Dorpat III, S. 59. 1889.

2) Dixson, Australian Medical Gazette, April 1887, S. 156. Ich babe die Resultate von $\mathrm{Dixs}$ on etwas ausführlicher angegeben, weil das Original nur schwer zugänglich ist, und weil sie von Stillmark (nach Husemann) unrichtig citirt sind. 
essig und Ammoniak und nachheriges Fällen des enthleiten Filtrates mit Alkohol. Dieser Niederschlag war farblos, flockig und sehr giftig, enthielt aber viel Eiweiss. Das Glykosid stellte er erweissfrei dar durch wiederholtes Fällen mit Chlorcaleium und Kali. Dieser Körper aber erwies sich als vollständig ungiftig und wurde nicht weiter untersucht.

Im Jahre 1889 wurde dann die unter Kobert's Leitung ausgeführte Arbeit von Stillmark veröffentlicht. Stillmark schreibt die giftige Wirkung der Ricinussamen einem Eiweisskörper zu, den er in verschiedener Weise zu isoliren versuchte. Am besten gelang die Fällung des Eiweisskörpers aus einem mit 10 procent. NaCl-Lösung bereiteten Auszuge dureh schwefelsaures Magnesium und schwefelsaures Natrium, hiernach wurden die Salze durch Dialyse entfernt. Dieser Körper, den Stillmark Ricin genannt hat; gehört seinen Reactionen nach zu der Gruppe der Globuline. Betreffs der Details der Reactionen, sowie der verschiedenen Darstellungsmethoden verweise ich auf die Originalarbeit.

Stillmark scheint keinen Versuch gemaeht zu haben, das Gift von dem Eiweiss zu trennen; er giebt nur dem Zweifel Ausdruek, ob er den Körper, der übrigens mit den giftigen Stoffen von Bubnow und Dixson identisch ist, den Globulinen oder Fermenten anreihen solle. Es ist jedoch möglich, dass das Ricin dem wahren Gifte nur als Verunreinigung beigemengt ist. Wenn nämlich irgend ein Gift sich in einer Eiweisslösung befindet, so wird es bekanntlich leicht mit jedem Eiweissniedersohlage mitgerissen und lässt sich dann nur sohwierig aus demselben auswaschen.

Während meines Aufenthaltes in Strassburg schlug mir Herr Professor Schmiedeberg im Jahre 1890 vor, diese Frage weiter zu verfolgen. Die nachstehenden Untersuchungen, die ich später in meinem Laboratorium in A n $n$ A r bor ergänzt habe, bieten wegen der Sohwierigkeit des Gegenstandes zwar nur wenige neue Thatsachen, die indessen für weitere Forschungen auf diesem Gebiete nicht ohne Bedeutung sein dürften; und obwohl meine Befunde bauptsächlich negative sind, so ist mir von versehiedenen Seiten nahe gelegt worden, dass es wegen weiterer Forschungen wünschenswerth sein möchte, meine Resultate zu veröffentlichen.

Anfangs benutzte ich die durch Extraction mit Alkohol und Aether vom Oel befreiten Ricinussamen zu meiner Untersuchung, später aber (ebenso wie Stillmark) die Ricinuspresskuchen, welche bei dem Auspressen des Oeles in den Fabriken übrig bleiben. 
Aus denselben lässt sich das Gift leicht durch Wasser oder Kochsalzlösung ausziehen. Ich unternahm zuerst, wie Dix son, die fractionirte Fällung mit Alkohol und fand, dass jeder Niederschlag. viel Eiweiss enthält und auch giftig ist. Die Niederschläge sind weder in Wasser, noch in Kochsalzlösung wieder vollständig löslich, und dies wiederholt sich bei jeder Fällung, bis am Ende sehr wenig lösliche Substanz übrig bleibt. Wiederholt habe ich eine giftige Lösung erhalten, die keine Biuretreaction gab; wenn sie aber durch Eindampfen concentrirt wurde, erschien beim Behandeln mit Kupfer und Kali die bekannte violette Farbe. Das Gift scheint also etwa dieselbe Löslichkeit in Alkohol zu haben, wie das Ricinuseiweiss. Keinen besseren Erfolg hatte ich, als ich mit Alkohol fällte, den Niederschlag mit Kali respective kohlensaurem Natrium auflöste, wieder mit Alkoboläther fällte und diese Procedur so lange wiederholte, bis nur sehr wenig lösliche Substanz übrig blieb. Solange die Lösungen giftig waren, enthielten sie auch Eiweiss. Dasselbe Resultat ergab sich, als ich die Niederschläge statt in Alkalien in Salzsäure löste. Versuche, in denen Alkalien und Salze der schweren Metalle angewendet wurden in der Absicht, eine Verbindung des Giftes mit schweren Metallen zu erhalten, wobei das Eiweiss durch die Alkalien in Lösung gehalten werden sollte, führten ebenso wenig zum Ziele. Wenn Eiweiss in Lösung blieb, so war die letztere giftig; sobald es vollständig verschwand, war keine Wirkung mehr zu erzielen. So wurden Kupferacetat, Bleiessig, Eisenchlorid und Quecksilbersublimat in Gegenwart von Alkalien nacheinander probirt.

Andere Fällungsmittel gaben kein besseres Resultat als der Alkohol. So konnte ich wiederholt alles Eiweiss durch Metaphospborsäure, Phosphorwolframsäure u. s. w. fällen, das Filtrat aber wurde unwirksam.

Bei dem Verfahren von Stillmark wurden die anorganischen Salze durch Dialyse entfernt. Bei Wiederholung seiner Versuche verdampfte ich das Wasser, von dem der Dialysator umspült war, und fand es giftig. Es enthielt aber Eiweiss und gab deutliche Biuretreaction.

Alle diese Versuche führten also zu dem gleichen Resultate: solange eine Lösung giftig ist, enthält sie anch Eiweiss, sobald letzteres entfernt ist, versehwindet auch die giftige Wirkung. Auf einen Punkt möchte ich spätere Forscher besonders aufmerksam machen. Die tödtliche Dosis ist so verschwindend klein, dass eine Lösung keine Biuretreaction zu geben braucht und dennoch giftig sein kann. Bedenkt man, dass für Kaninchen eine Gabe von $0,04 \mathrm{mg}$ 
Ricin (!) pro kg Körpergewicht tödtlich ist, so können schon 2 ccm einer Lösung von 1 : 50000 den Tod herbeiführen. Bei dieser Verdünnung ist aber die Biuretreaction nicht mehr deutlich, und die meisten anderen Eiweissreactionen bleiben auch zweifelhaft. WiederLolt habe ich geglaubt, eine giftige eiweissfreie Lösung in Händen zu haben, wenn dieselbe aber durch Eindampfen oder Fällen mit Alkoholäther concentrirt wurde, zeigte sie wieder die Biuretreaction, also die Anwesenheit von Proteïden.

Ich kam daher zu dem Schlusse, dass das Ricinusgift sich von den Eiweissstoffen des Samens nicht trennen lässt, sondern entweder selbst ein Eiweisskörper ist oder wenigstens mit dem Eiweisse in einer Verbindung sich befindet, aus der es durch die gewöhnlichen Methoden nicht frei zu machen ist.

Schon Pecholier ${ }^{1}$ ) hat gemeint, dass bei der Wirkung des Ricinusgiftes ein fermentativer Process im Gange ist, und Stillmark bat sich auch dieser Ansicht angeschlossen. Indessen konnte er durch das Verhalten des Ricins gegenüber verschiedenen Agentien keine sicheren Beweise dafür beibringen. Er stützt seine Ansicht auf die Wirkung des Ricins auf das Blnt. Immerhin bleiben noch einige Thatsachen übrig, die auf eine fermentartige Beschaffenheit des Ricins hinweisen könnten. Die wichtigsten derselben sind, nach meiner Ansicht, die minimale Dosis letalis und der lange Zeitraum zwischen der Giftapplication und der Wirkung. Die tödtliche Wirkung des Ricins übertrifft die aller bekannten Gifte. Wie oben angegeben, ist $0,04 \mathrm{mg}$ pro $\mathrm{kg}$ Kaninehen tödtlich, wäbrend das Cobragift ${ }^{2}$ ) ein anderes "Toxalbumin", das dem Ricin am nächsten steht, $0,079 \mathrm{mg}$ pro $\mathrm{kg}$ Körpergewieht erfordert. Indessen sind einige andere Gifte bekannt, deren Toxicität derjenigen des Ricins zwar nicht gleich ist, doch nahe steht. So zum Beispiel ist die letale Menge des Aconitins bei Kaninchen etwa $0,1-0,2 \mathrm{mg}$ pro $\mathrm{kg}$ Körpergewicht, während dieselbe bei Mensehen noch bedeutend kleiner pro kg ist. Die Latenzzeit des Ricins ist auch sehr merkwürdig. Ich habe wiederholt die Beobachtung von Still mark bestätigen können, dass Kaninchen vier, fünf oder noch mehr Tage augenscheinlich vollkommen normal bleiben und am Ende dieser Zeit plötzlich sterben, und die typischen postmortalen Erscheinungen der Rieinusvergiftung: zeigen. Frösche bleiben häufig allem Anscheine nach noch länger

1) Etude sur l'empoissonnement par les semenes du Ricin 1869 (citirt nach nach Stillmark).

2) Martin, Proc. Roy. Soc: XLVI, p. 108. 
normal, bevor sie endlich zu Grunde gehen. Indessen besitzen andere Gifte, z. B. das albuminsaure Kupfer ${ }^{1}$ ), sowie das Zinn ${ }^{2}$, auch cine ungemein lange Latenzzeit.

Es schien mir doch möglich, dass das Ricinusgift im Blute entwede $r$ selbst vermehrt wird oder durch eine fermentative Thätigkeit ein neues Gift darin erzeugt. Um dies zu prüfen, habe ich einig'; Versuche angestellt, in clenen eine bekannte Menge des Ricinus; ;iftes mit verschiedenen Eiweisslösungen gemischt und einige Zeit stehen gelassen wurde. Nach verschieden langer Zeit wurde die G.ftigkeit der Lösung untersucht. So z. B. habe ich $10 \mathrm{ccm}$ einer Eiereiweisslösung mit $3 \mathrm{ecm}$ einer Ricinlösung, von der $1 \mathrm{cem}$ tödtlich wirkte, 10 Tage bei Zimmertemperatur gehalten. Am Ende dieser Zeit waren $3 \mathrm{ccm}$ der Lösung ungiftig, während $5 \mathrm{ccm}$ schnell den Tod herbeiführten. Es scheint also, dass keine Zunahme der Giftigkeit stattgefunden hatte. Andere Versuche mit angesäuertem Eiereiweiss, mit Blutserum, Serumalbumin, Serumglobulin, wobei verschiedene Temperaturen angewendet wurden, gaben dasselbe negative Resultat.

Es war aber möglich, dass im lebenden Thiere dennoch eine Vermehrung der Giftigkeit eintritt. Ich habe deshalb ein Kaninchen entblutet, 2 Tage nachdem eine giftige Menge Ricin in eine Vene injicirt worden war. Das Blut wurde dann defibrinirt und einem kleineren Kaninchen transfundirt, das aber ganz normal blieb. In anderen Fällen wurden 2 Kaninchen mit Ricin getödtet und das Blut, soweit das möglich war, aus dem Herzen und den grossen Gefässen entleert und in die Vene eines anderen Thieres eingespritzt. Das Thier blieb aber vollkommen gesund. Nur in einem Versuche, in welchem dem Blute das Exsudat der Bauchhöhle beigemengt war, starb das zweite Thier nach einigen Stunden, zeigte aber keine der charakteristischen Merkmale der Ricinusvergiftung bei der Autopsie.

Bei Fröschen bekam ich ein verschiedenes Resultat. Bei diesen Thieren findet man, ausser den Zeichen einer intensiven Reizung zu Anfange der Vergiftung, nach dem Tode regelmässig einen Bluterguss in den Magen. Wenn der blutige Mageninhalt einem zweiten Frosch subcutan injicirt wird, so stirbt auch dieses Thier unten den gleichen Erscheinungen. Der Mageninhalt ist wieder giftig, und eine ganze Reihe von Fröschen kann auf diese Weise nacheinander ver-

1) Schwarz, Dies. Archiv. XXXV, S, 437. 1895.

2) Vergl. White, Arch. f. exp. Path. u. Pharm. Bd. XIII, S. 53, 1881. 
giftet werden. Es ist aber schwierig; die letale Menge bei Fröschen $\mathrm{zu}$ bestimmen, und ich bin nicht geneigt, viel Gewicht auf diese Versuche zu legen. A $t{ }^{1}$ ) hat Schlangengift und Choleragift nach der Vergiftung von Thieren im Magen wiedergefunden, und es scheint, dass auch das Ricin, wenigstens bei Fröschen, hier ausgeschieden wird.

Nach meinen Versuchen ergaben sich also keine Gründe, anzunehmen, dass das Ricinusgift innerhalb oder ausserhalb des Körpers sich zu vermehren oder andere giftige Substanzen zu erzeugen im stande sei. Immerhin ist es möglich, dass in den festen Geweben solche Substanzen entstehen, indessen haben wir bis jetzt, ausser der Blutwirkung, keine Gründe, dem Ricinusgift eine fermentative Wirkung zuzusehreiben oder es uns als eine von den anderen Eiweisskörpern, ausser durch seine Giftigkeit, abweichende Substanz vorzustellen.

Es war daher von Interesse, diesen Körper in dieser Richtung näher zu untersuchen. Ricin wurde von Stillmark durch Fällen des 10 proc. Kochsalzauszuges mit schwefelsaurem Magnesium und Natron bereitet. Die Reactionen des Ricins mit den gewöhnlichen chemischen Agentien sind sohon von Stillmark angegeben worden, dessen Beschreibung ich durchaus bestätigen kann. Nur fand ich, dass es mit schwefelsaurem Magnesium quantitativ gefällt wurde, wenn die Lösung durch 24 stïndiges Schütteln mit dem Salze vollständig gesättigt wurde, und dass es darum der Gruppe der Globuline einzureihen ist. Um vollkommen reines Ricin zu erhalten, fällte ich einen Auszug der Ricinuspresskuchen mit Magnesiumsulfat, löste den Niederschlag; soweit es ging, durch Entfernen der Salze mittelst Dialyse, filtrirte, fällte wieder mit Magnesiumsulfat und löste wieder in derselben Weise. So wurde eine gelbliche Lösung erhalten, die, neben Salzen, ausser Ricin nur wenig organische Substanzen zu enthalten schien. Durch Zusatz von etwas Thymol und Kaltstellen konnte diese Lösung monatelang ohne Zersetzung aufbewahrt werden.

Die Coagulationstemperatur des Ricins fand ich sehr verschieden. Wenn eine Lösung schnell erhitzt wird, bedarf es einer ziemlich bohen Temperatur, um das Globulin zum Gerinnen zu bringen, wälrend bei langsam steigender Temperatur ein Coagulum bei viel niedrigerer Temperatur ausfällt. Wenn zum Beispiel die Temperatur in 35 Secunden um $1^{0}$ anstieg, so wurde die Flüssigkeit bei $63-$ $64^{\circ}$ trüb, und bei $74^{0}$ bildeten sich Flocken, während wenn die Tem-

1) Deutsch. Med. Wochenschrift 1892. S. 954. 
peratur nur um $1^{0}$ in 10 Minuten zunahm, Opalescenz und Flocken schon bei $55^{0}$ erschienen. Bei sehr langsamem Erhitzen kann man sogar bei $51^{0}$ ein Coagulum erzeugen. Als eine Lösung bei $58^{\circ}$ gehalten wurde, fielen Niederschläge tagelang aus. So wurde in einem Falle eine Lösung 96 Stunden bei $58^{\circ}$ im Trockenschranke gehalten, dann filtrirt und wieder auf $58^{\circ}$ gebracht. Nach 24 Stunden entstand wieder ein Niederschlag. Der Lösung wurde vorsichtig Wasser alle paar Stunden zugesetzt, so dass keine Concentration derselben stattfand. Wenn die Lösungen etwas angesäuert waren, hatten sie keine constantere Coagulationstemperatur.

Diese Variabilität könnte durch die Annahme erklärt werden, dass mehrere Globuline in dem Riein vorhanden sind. Indessen scheint das nicht der Fall zu sein, wie durch den folgenden Versuch gezeigt wurde. Eine Lösung, von der $1 / 260 \mathrm{ccm}$ pro kg Körpergewicht giftig war, wurde bei $54^{0} 60$ Stunden constant gehalten und dann filtrirt. Es ergab sich, dass das Filtrat 1/6 weniger organische Substanz (d. h. Ricin) enthielt als im Anfange, und dass 1/200 $\mathrm{ccm}$ nöthig war, den Tod herbeizuführen. Wenn dem Ricin die Giftwirkung zukommt, so hätte das $(6 / 5 \succ 1 / 260 \Rightarrow 1 / 21 \mathrm{ocm}$ als giftige Dosis erfordert. Die kleine Differenz liegt innerhalb der Fehlergrenzen. Es seheint daher, dass das Ricin (Ricinglobulin) ein einbeitlicher Körper ist, oder wenigstens, wenn mehrere Globuline darin vorhanden sind, sie dieselbe Giftigkeit besitzen.

Dieser Versuch ist natürlich auch eine starke Stütze für die Annahme, dass Ricin und Ricinusgift identisch sind. Die Abnabme der Toxicität des Ricins, wenn Lösungen desselben erhitzt werden, kann durch das Unlöslichwerden erklärt werden und braucht nicht die Annahme einer fermentativen 'Thätigkeit zu sein, wie Stillmark meint.

Wenn alles Ricin ausgefällt wird, was sicher beim Kochen, häuffig aber schon bei $80^{\circ}$ erreicht werden kann, so verliert die Lösung: alle giftige Wirkung. Ricinuslösungen können aber erhitzt werden, ohne ihre Giftigkeit vollständig zu verlieren, indessen sobald ein Coagulum entsteht, wird die Giftigkeit proportional vermindert.

Eine der merkwtirdigen Eigenschaften des Ricins ist, dass es mit allen Niedersohlägen besonders mit anderen Proteïden niederfällt. Wenn zum Beispiel eine Lösung des Ricins, durch die ein Kohlensäurestrom stundenlang durehgeleitet worden war, ohne einen Niederschlag zu erzeugen, zu Blutserum zugesetzt und dann Kohlensäure durchgeleitet wurde, bis ein Niederschlag entstand, so fand ioh diesen sehr giftig, sogar nachdem er lange ausgewasohen 
war. Eine Ricinlösung, die schon mit Salzsäure ausgefällt worden war und mit dieser Säure keine weitere Trübung gab, wurde einer Eiereiweisslösung zugesetzt. In dieser erzeugte die Salzsäure einen. Niederschlag des Eiereiweisses, der stark giftig war. Die Anwesenheit des Ricins kann natürlich viel sicherer nachgewiesen werden, als die anderer Eiweisskörper, und es ist möglich, dass diese Eigenschaft des Anhaftens allen Proteïden gemeinsam ist. Sollte das der Fall sein, so ist die Isolirung von reinen Proteïden sicher noch schwieriger, als gewöhnlich angenommen wird.

Nicht nur an löslichem Eiweisse klebt das Ricin, sondern auch an geronnenen Körpern. So fand ich, dass wenn man Fibrinstücke in Ricinlösungen einige Zeit liegen lässt, das Gift durch Auswaschen mit Wasser und Kochsalzlösung bis zum Verschwinden der Proteïdreactionen im Waschwasser nicht mebr entfernt wird, indem beim Waschen mit einer Sodalösung diese noch giftig wurde.

Auch an anderen Niederschlägen haftet das Ricin fest. Wenn Barytlösung, mit Ricinlösung gemischt, ein etwaiger Niederschlag abfiltrirt und dann der Baryt durch Kohlensäure ausgefällt wurde, so enthielt der Baryumcarbonatniederschlag viel Ricin. Tichomir off ${ }^{1}$ ) erwähnt, dass Ricin in saurer Lösung durch alkalische Nuclë̈nsäurelösungen gefällt wird. Die saure Ricinlösung erzeugt, wie zu erwarten war, einen Niederschlag von Nuclë̈nsäure, und dieser reisst das Ricin mit. Etwas Specifisches für die Nucleïnsäure giebt es hier nicht. Unter denselben Bedingungen habe ich einen giftigen Niederschlag mit benzoësaurem Natrium erhalten.

Das Anhaften des Ricins an allen möglichen Proteidniederschlägen habe ich so häufig constatirt, dass ich nicht wenig überraseht war, einen zweiten Eiweisskörper in Ricinus zu finden, der keine giftige Wirkung besitzt. Er bleibt in der Lösung, nachdem Ricin mit schwefelsaurem Magnesium ausgefällt worden ist, und scheint zu den Albumosen zu gehören. Um ihn darzustellen, habe ich den Rieinusauszug mit gepulvertem Magnesiumsulfat 24 Stunden in einer "Schuittelmaschine" geschüttelt, dann abfiltrirt und wieder mit Magnesiumsulfat geschüttelt, bis ich sicher war, dass alles Ricin ausgefällt war. Es bleibt dann die Albumose in der gesättigten Lösung, aus welcher sie durch Sättigen mit schwefelsaurem Ammoniak ausgefällt werden kann. Es ist aber schwierig, die Albumose von den Salzen zu befreien, indem sie in bedeutender Menge dialysirt. Ioh habe sie daher durch Salzsäure gefällt, was in ge-

1) Zeitschrift für physiologische Chemie Bd. XXI, S. 90 . 
sättigten Lösungen von schwefelsaurem Magnesium gut gelingt, obschon Salzsäure eine Trübung und keinen Niederschlag erzeugt, wenn Salze nicht im Ueberschusse vorhanden sind. Häufig aber konnte ich nicht alle Albumose mit Salzsäure fällen, während das schwefelsaure Ammoniak sie quantitativ zu fällen scheint. Der Niederschlag dureh Salzsäure wurde abfiltrirt und in Wasser gelöst. Bei dieser Methode muss man vorsichtig sein. Man darf nicht zu viel Salzsäure zusetzen und auch den Niederschlag nicht mit Wasser auswaschen, indem die Albumose in tiberschüssiger Salzsäure und in Wasser löslich ist. Die Albumose kann auch durch Kochen einer Ricinuslösung bereitet werden, wobei das Ricin gerinnt und abfiltrirt werden kann. Es bleibt dann die Albumose in der Lösung, woraus sie dureh Alkohol ausgefällt wird. Die Albumose ist vollständig ungiftig und hat keine solche Wirkung auf die rothen Blutkörperchen, wie sie Stillmark für das Ricin beschreibt. Sie ist in Wasser leicht löslich, gerinnt nicht durch Kochen, wird durch Alkoholäther, und durch Salz-, Salpeter- und Schwefelsäure in gesättigten Lösungen von neutralen Salzen gefällt. Schwefelsaures Ammoniak fällt sie. Die Biuretreaction wird schon in der Kälte erzeugt, und sie giebt die gewöhnliche Proteïdreactionen mit Millon's Reagens, Phosphorwolframsäure, Pikrinsäure, Quecksilberchlorid, Blutlaugensalz mit Essigsäure u. s. w. Sie geht durch das Dialysatorpapier jedoch etwas langsam. Dass man die Albumose frei von dem Gifte erhalten kann, scheint darauf hinzudeuten, dass das Ricin nicht gelösten Proteïden anhaftet, sondern nur mit den Niederschiägen mitgerissen wird.

Stillmark hat eine merkwürdige Wirkung des Ricins auf die rothen Blutkörperchen beschrieben. Der Zusatz einer minimalen Menge Ricin zum Blute genügt, die Blutkörperchen derart zu verändern, dasssie nicht mehr im Serum oder im Plasma schwimmen, sondern in kleinen zusammenhängenden Massen zu Boden sinken. Diese Wirkung nehmen Stillmark und Kobert als eine der natuirlichen Blutgerinnung etwas ähnlichen Vorgang an und schreiben dem Ricin eine fibrinfermentartige Wirkung zu. Ich kann das Vorkommen dieser Wirkung vollkommen bestätigen, aber es schien mir, dass dieselbe und die natürliche Blutgerinnung. ganz verschiedener Natur sind. Bei der mikroskopischen Beobachtung des Vorganges schien es vielmehr, als ob die Körperchen durch irgend einen klebrigen Niederschlag zusammengekittet wären. Ich hätte diese Wirkung weiter verfolgt, wäre ich nicht überzeugt, dass die tödtliche 
Wirkung von derselben ganz unabbängig ist. Nach Stillmark sollen die Aggregate von Blutkörperchen die Capillaren, besonders die der Darmgefässe verstopfen und auf diese Weise Thrombosen und Ekchymosen bewirken, wovon Erosionen und Ulcera der Darmsohleimhaut und des Magens die natürliche Folge sind.

Flexner ${ }^{1}$ ) aber, der die postmortalen Erscheinungen genauer untersucht hat, meint, dass die Hauptwirkung des Ricins in der Zellennekrose der Leber und der Lymphdrüsen besteht, und dass die Blutergïsse und die anderen Erscheinungen von dieser Wirkung abhängig sind.

Ich hatte mich schon vor dem Erscheinen der letztgenannten Arbeit überzeugt, dass die Blutwirkung nur von nebensäehlichem Interesse ist. Wenn man nämlich verhältnissmässig grosse Mengen Ricin in eine Vene einspritzt und das Thier nachher verbluten lässt, so zeigen die rothen Blutkörperchen nichts von diesem abnormen Verhalten, bis Riein dem Blute in vitro zugesetzt wird. Einen noch sichereren Beweis erhielt ich dureh die Blutuntersuchung eines immunen Thieres. Ehrlich ${ }^{2}$ ) hat nämlich gezeigt, dass ein Thier durch Füttern mit Ricin in kurzer Zeit Immunität gegen die Ricinwirkung erlangt. Ich habe ein Kaninchen so "ricinfest" gemacht, dass eine $5000 \mathrm{mal}$ so grosse Menge als die, welche ein normales Thier sicher tödtet, bei ihm ganz ohne Wirkung blieb. Sein Blut aber zeigte die Ricinreaction ganz wie das eines normalen Kaninchens, nur dass es noch etwas empfindlicher schien. Gegen diese Blutwirkung also nützt die Immunität nichts.

Stillmark verwirft die alte Angabe der Reizwirkung des Rieinusgiftes. Dass das aber nicht mit Recht geschieht, zeigt die von Ehrlich beobachtete entzündungserregende Wirkung am Auge, sowie die fast regelmässig auftretende Entzündung und Nekrose bei subcutaner Injection. Die tödtliche Wirkung des Rieinusgiftes ist wahrscheinlich mit dieser Reizwirkung eng verbunden; wenigstens kann ich nach meinen Versuchen mit ricinfesten Thieren behaupten, dass die Giftwirkung mit der Blutwirkung nichts zu thun hat. Es fehlt überhaupt noch an Gründen für die Annahme, dass diese Wirkung auf das Blut in irgend welchem Maasse im Thierkörper stattfindet. Bis jetzt ist ihr Vorkommen nur an dem todten Blute im Reagenscylinder sicher festgestellt.

1) The Medical News, August 14, 1894.

2) Deutsche Med. Wochenschr. 1891. S. 976. 